\title{
Trapping of Image-Potential Resonances on a Free-Electron-like Surface
}

\author{
M. Winter, ${ }^{1}$ E. V. Chulkov, ${ }^{2,3}$ and U. Höfer ${ }^{1,2}$ \\ ${ }^{1}$ Fachbereich Physik und Zentrum für Materialwissenschaften, Philipps-Universität, 35032 Marburg, Germany \\ ${ }^{2}$ Donostia International Physics Center (DIPC), 20018 San Sebastián, Spain \\ ${ }^{3}$ Departamento de Física de Materiales, UPV/EHU and CFM-MPC, Centro Mixto CSIC-UPV/EHU, 20080 San Sebastián, Spain
}

(Received 8 September 2011; published 28 November 2011)

\begin{abstract}
Image-potential states have been studied by two-photon photoemission for the surface of $\mathrm{Al}(100)$ where the whole series is energetically degenerate with free-electron-like bulk states. In contrast with expectations, the series of resonances is not smeared out to one broad structure as a result of a strong coupling to the bulk continuum. Instead, the first resonance $(n=1)$ is found to be suppressed, and the resonances with quantum numbers $n=2,3,4,5$ are resolved as individual peaks in the time-resolved spectra. Both effects are suggested to be a consequence of resonance trapping.
\end{abstract}

DOI: 10.1103/PhysRevLett.107.236801

PACS numbers: 73.20. $-\mathrm{r}, 78.47 .-\mathrm{p}, 79.60 . \mathrm{Bm}$

Electron transfer processes at solid surfaces and interfaces play a decisive role in many fields of research, such as surface chemistry or nanoscience. In the past decade, experimental and theoretical studies of the electronic decay at metal surfaces have considerably advanced the microscopic understanding of those inelastic scattering phenomena that drive the transfer in the case of states located in the forbidden energy gap of the solid [1,2]. Many practical situations, however, involve energy levels, e.g., of adatoms, molecules, clusters, or small islands, that are resonant with bulk bands. Then, elastic transfer channels are expected to dominate over the inelastic ones [3-5]. Interestingly, this rather basic, resonant electron transfer appears to be less well-understood than some of the inelastic electron-electron or electron-phonon many-body decay processes. Generally, when a quantum system is embedded in a continuum, the coupling can lead to striking effects like Fano-type interference phenomena in the case of a single level or avoided overlap and trapping in the case of two or more adjacent levels. Whereas such phenomena that go far beyond a simple resonance broadening have long been investigated intensively in atomic and nuclear physics [6-9], they are usually not taken into account in the discussion of electron decay at surfaces.

The investigation of image-potential resonances of $\mathrm{Al}$ (100) by time-resolved two-photon photoemission (2PPE), reported in this Letter, clearly demonstrates the importance of such effects in surface physics. Like the well-known image-potential states in the projected band gap [10-14], image-potential resonances form a Rydberg series of hydrogenlike states below the vacuum level $[15,16]$. In contrast to gap states, electrons excited into these resonances are not confined to the surface, but can propagate into the metal without the need to undergo collisions. Even for surfaces with a wide projected band gap close to the vacuum level, such as $\mathrm{Cu}(111)$ or $\operatorname{Ag}(111)$, it has been suggested that they delocalize faster in this way than they decay by electron-hole-pair excitation $[12-14,16]$. In the case of the $\mathrm{Al}(100)$ surface investigated here, the corresponding energy gap is relatively narrow and located far below the vacuum level [Fig. 1(a)]. In such a situation, one expects the coupling of the hydrogenic levels to the continuum to be so strong that the resulting widths of the resonant states become comparable to their energy spacing. For the extreme case of a jellium surface, $a b$ initio calculations predict one single maximum in the surface density of states, peaked at the position of the first imagepotential state [17].

In contrast, our 2PPE spectra, recorded at different pump-probe delay times, reveal individual peaks in the intensity. Whereas we are able to unambiguously assign these maxima to the quantum numbers $n=2,3,4,5$ of the Rydberg series, surprisingly, the data show no signature of the first resonance $(n=1)$. Model calculations with a series of discrete levels coupled to a structureless continuum suggest that both the suppression of the first and the observability of clearly resolved higher resonances are a general phenomenon, called resonance trapping, that will generally occur for sufficiently strong continuum coupling of two or more electronic states at surfaces. In the time domain, it manifests itself in a bi-exponential decay of the 2PPE intensity.

The experiments were carried out in a UHV chamber with a base pressure of $4 \times 10^{-11}$ mbar. The $\mathrm{Al}(100)$ sample was cleaned by repeated argon sputtering $\left(2 \mu \mathrm{A} / \mathrm{cm}^{2}, 15 \mathrm{~min}\right)$ and annealing $\left(400{ }^{\circ} \mathrm{C}, 45 \mathrm{~min}\right)$ cycles and regularly checked for cleanliness and structural order by x-ray photoelectron spectroscopy and low-energy electron diffraction, respectively. The 2PPE measurements were performed at room temperature with the $p$-polarized infrared (IR) and third harmonic ultraviolet (UV) pulses of a Ti:sapphire oscillator, with photon energies of $1.55 \mathrm{eV}$ and $4.65 \mathrm{eV}$ and pulse duration of $43 \mathrm{fs}$ and $66 \mathrm{fs}$, respectively. The photoelectrons were recorded by a hemispherical analyzer with $25 \mathrm{meV}$ energy and $1.2^{\circ}$ angular resolution. The angle between the detected photoelectrons 

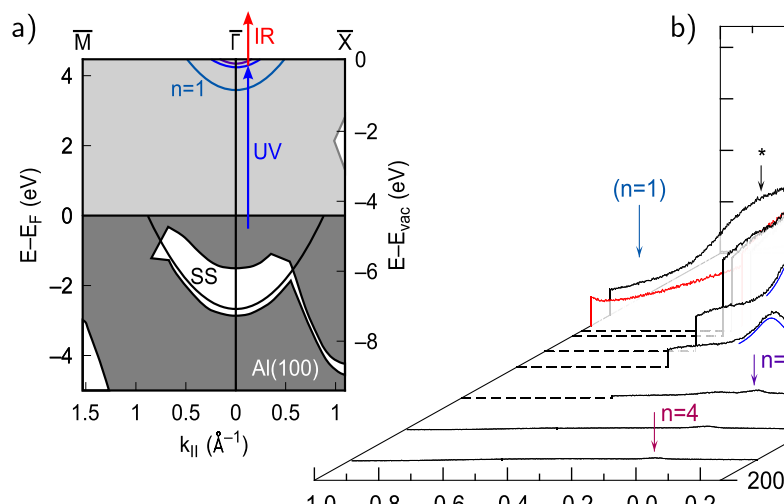

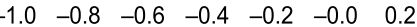

Energy $\mathrm{E}-\mathrm{E}_{\mathrm{vac}}(\mathrm{eV})$

b)

b)

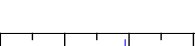

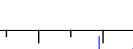

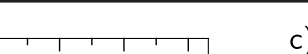

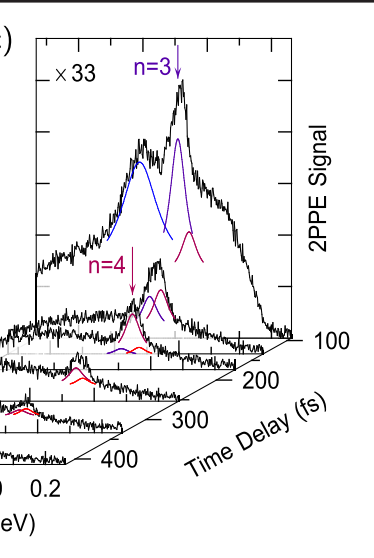

FIG. 1 (color online). (a) Schematic drawing of the electronic structure of $\operatorname{Al}(100)$ and the 2PPE excitation scheme. Grey shaded areas denote projected bulk bands [30], parabolic lines the Shockley surface state (SS) and the first two image-potential resonances $(n=1, n=2)$. (b),(c) 2 PPE spectra recorded with different delay times between the UV excitation and the IR probe pulses. Lorentzian lines and arrows indicate the contributions of individual resonances to the total spectra. As distinct from the resonances, the feature marked (*) is excited by IR and probed by UV pulses. The energy scale in (b) is not applicable to this 2PPE process. The feature $(*)$ originates from an intermediate state $0.9 \mathrm{eV}$ above the Fermi level (see text).

and the incident laser light was $75^{\circ}$. Energies are measured relative to the Fermi level $E_{F}$ but will be referred to the vacuum energy $E_{\mathrm{vac}}=E_{F}+\Phi$ in the following. The work function $\Phi$ of the sample was determined to be $(4.54 \pm$ $0.05) \mathrm{eV}$.

Figures 1(b) and 1(c) display 2PPE spectra taken at different time delays of the IR probe pulses relative to the UV excitation pulses. For zero delay [emphasized spectrum in Fig. 1(b)], the data exhibit a clearly visible peak at an energy of $210 \pm 10 \mathrm{meV}$ below the vacuum level and a broad structure at higher energies that extends to $\sim 100 \mathrm{meV}$ above $E_{\mathrm{vac}}$. With increasing time delay, additional peaks at higher energies (i.e., lower binding energies) can clearly be resolved in the spectra, in particular, a peak at $-91 \pm 6 \mathrm{meV}$ [Fig. $1(\mathrm{c}), \Delta t=100 \mathrm{fs}$, a peak at $-57 \pm 8 \mathrm{meV}$ [Fig. 1(c), $\Delta t=200 \mathrm{fs}$ ], and finally a very weak peak at $-36 \pm 9 \mathrm{meV}$ at the large delays. We identify these peaks with the image-potential resonances $n=2,3,4,5$. The measured energies of these resonances agree well with the Rydberg formula $E_{n}=-0.85 \mathrm{eV} / n^{2}$ (Fig. 2).

Most remarkably, neither the 2PPE spectra plotted in Fig. 1 nor any of the other recorded 2PPE spectra showed a structure that we could assign to the first image-potential resonance $(n=1)$. Some data exhibit a shoulder on the low-energy side of the $(n=2)$ peak. An example is the spectrum in Fig. 1(b) corresponding to a small negative delay, i.e., the IR pulse preceding the UV pulse. This feature, however, is not representative for well-prepared $\mathrm{Al}(100)$ and has no relationship to the image-potential resonances. It is pumped by IR and probed by UV pulses and located $\sim 0.9 \mathrm{eV}$ above the Fermi level and only shows up at a similar final-state energy as the image-potential resonances for small pump-probe delays. We believe that it is due to small residual oxygen contaminations or to defects on the surface.

The $(n=1)$ peak is usually the most pronounced feature in the spectra of image-potential states [10-14]. A reason why it does not appear in the 2PPE spectra of $\mathrm{Al}(100)$ could be that there are no suitable initial states from where it is populated by the $4.65-\mathrm{eV} \mathrm{UV} \mathrm{pulse.} \mathrm{This} \mathrm{is,} \mathrm{however,}$ not compatible with the measured $\mathrm{Al}$ band structure $[18,19]$. Moreover, if the projected band gap at the $\bar{\Gamma}$ point was closer to the Fermi level than indicated in Fig. 1(a), then also the defect-induced state at $0.9 \mathrm{eV}$ above the Fermi level could not be populated by the $1.55-\mathrm{eV}$ IR pulse. We therefore exclude the possibility of missing initial states as a possible origin of the missing $(n=1)$ resonance. In the

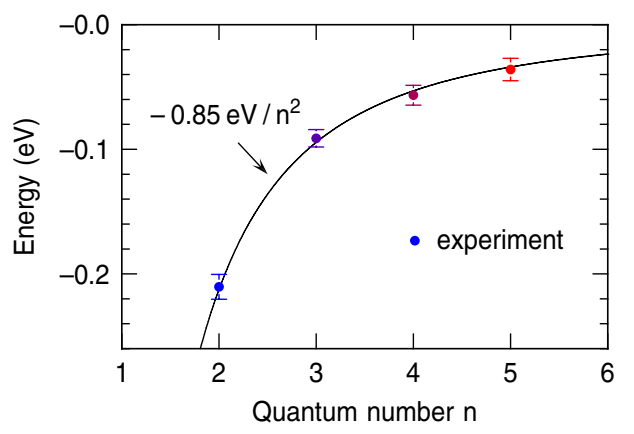

FIG. 2 (color online). Energy positions of image-potential resonances as functions of quantum numbers $n$. The experimental values (filled circles) were determined by fitting Lorentzian line shapes to several 2PPE spectra (5-13 for each $n$ ). The error bars indicate the uncertainties of determining these peak positions relative to each other and do not include a possible overall shift of the energy scale by $\pm 50 \mathrm{meV}$ from the error of the sample work function. 
following, we show that its suppression is a direct consequence of the strong coupling of the whole series of resonances to the continuum.

In terms of a basic quantum mechanical description, the image-potential resonances can be viewed as a series of discrete levels $|n\rangle$ with energies $E_{n}=-0.85 \mathrm{eV} / n^{2}$ that are coupled to a structureless continuum of equidistant states. The coupling strengths $V_{n \nu}$, which enter as offdiagonal elements $\langle\nu|H| n\rangle$ in the Hamiltonian of the combined system, will then be independent of $|\nu\rangle$. For the $n$ dependence, we choose $\left|V_{n \nu}\right|^{2} \propto n^{-3}$, which is the limiting behavior for large $n$ [15].

Figure 3 shows the density of states that results from the numerical diagonalization of the Hamiltonian of the coupled system for different coupling strengths. The latter is denoted in terms of the linewidth $\Gamma_{1}$ of the (hypothetical) isolated first level. While a single level would simply broaden with increasing coupling, the situation becomes more complex for two or more states. For the case of two equally strongly coupled levels, it is easy to show analytically that they approach each other until they join to one trapped resonance as soon as the coupling strength $\Gamma$ reaches the level spacing $\Delta$. With further increasing coupling, the apparent width of the resonance then becomes smaller again, a phenomenon called resonance trapping or resonance narrowing [9]. For different coupling strengths, the maximum of the trapped resonance is no longer located in the middle of the two levels but closer to the more weakly coupled one. In the case of the Rydberg series of Fig. 3, a reminiscence of this behavior can be seen for $n=2$. This resonance broadens and slightly shifts to lower energy with increasing coupling strength. But, even before this shift becomes significant, for coupling strengths $\Gamma_{1} \geq$ $0.5 \mathrm{eV} \simeq \Delta_{12}$, the broadening of the $(n=1)$ resonance has become so strong and its amplitude so small that it is hardly

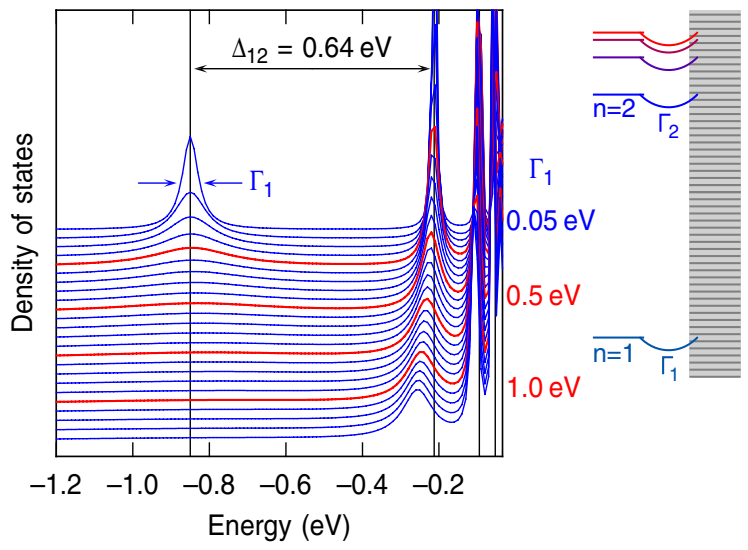

FIG. 3 (color online). Density of states calculated for a discrete Rydberg series $-0.85 \mathrm{eV} / n^{2}$ coupled to an unstructured continuum. The coupling strength is indicated in terms of the width $\Gamma_{1}$ of the $(n=1)$ resonance (see text). It increases in steps of $0.05 \mathrm{eV}$ from top to bottom. visible as a peak. From the comparison of these calculations with the experimental data [20], we thus conclude on a large coupling strength $\Gamma_{n}$ of the order of the level spacing $\Delta_{n, n+1}$ for the $\mathrm{Al}(100)$ image-potential resonances, i.e., $\Gamma_{1} \geq 0.5 \mathrm{eV}$. Surprisingly, this strong coupling does not wash out the peak structure of the Rydberg series for $n \geq 2$ but leads to the disappearance of the $(n=1)$ resonance. The $(n=1)$ resonance becomes the open channel of the series, whereas the other levels become trapped states.

An independent experimental verification of the large value of the coupling strengths $\Gamma_{n}$ can be obtained by analyzing the decay of the 2PPE intensity as a function of pump-probe delay. Figure 4 shows data recorded near the energy maxima of the resonances. The decay of the $(n=2)$ resonance at $-0.2 \mathrm{eV}$ is hardly distinguishable from the cross correlation of the two laser pulses. The best fit to a rate equation model would give a decay time of $9 \pm 6$ fs. The signals at higher energies show a similarly fast initial decay that is then followed by a decay on a slower time scale. Superimposed on that, the 2PPE intensities exhibit weak quantum-beat oscillations as functions of the delay time [11].

For the analysis of these data, we have performed density matrix calculations of the complete $2 \mathrm{PPE}$ process, including resonance trapping [22]. Similar to the model discussed above, the relative continuum coupling of each level $n$ is initially set to scale with the level spacing $\Delta_{n, n+1}$. Diagonalization of the combined system, Rydberg series plus continuum, then changes these ratios depending on the magnitude of the overall coupling strength. The latter is again denoted in terms of the linewidth $\Gamma_{1}$ of the first level.

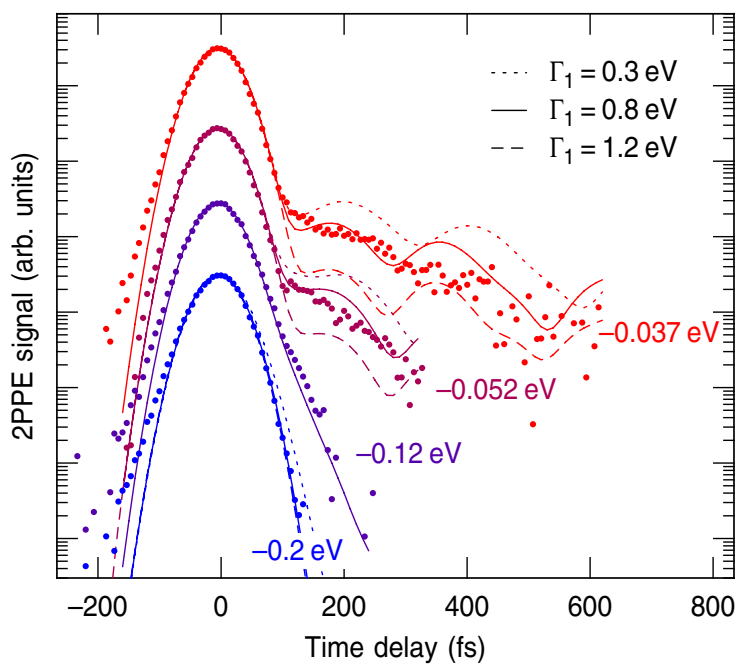

FIG. 4 (color online). 2PPE intensity from $\mathrm{Al}(100)$ as a function of delay time between UV pump and IR probe pulses corresponding to different intermediate state energies (filled circles) and results of corresponding density matrix simulations for different coupling strength (lines). The data have been normalized for equal maximum intensity and offset for clarity. 
$\Gamma_{1}$ is thus the only adjustable parameter to describe the whole data set. This model leads to a satisfactory description of the experimental results for coupling strengths $\Gamma_{1}=0.8 \pm 0.3 \mathrm{eV}$ (Fig. 4), i.e., values of $\Gamma_{1}$ for which the calculated density of states also begins to show significant effects of resonance trapping. Simulations with larger $\Gamma_{1}$ 's suppress the relative signal intensity of the slowly decaying contribution at low binding energies too much (Fig. 4, dashed line). Weaker coupling results in too slow a decay of the $(n=2)$ resonance (Fig. 4, dotted line).

The coupling strength $\Gamma_{1}=0.8 \mathrm{eV}$ corresponds to a lifetime of $\tau_{1}=\hbar / \Gamma_{1} \simeq 0.8 \mathrm{fs}$. It is interesting to compare the measured lifetime of the higher resonances with $\tau_{n}=$ $\hbar / \Gamma_{1} \times n^{3} \simeq 0.8$ fs $\times n^{3}$ as derived purely from their continuum coupling. They are consistently found to be longer. For $n=4$, e.g., $\tau_{4} \simeq 50 \mathrm{fs}$, whereas the experimental lifetime of the slowly decaying component (Fig. 4, $-0.052-\mathrm{eV}$ data, delay $>100 \mathrm{fs}$ ) is $85 \pm 15 \mathrm{fs}$. Like the spectral resolution of the resonances, this comparison indicates that electrons excited into one of the higher members of the series start to get trapped and live longer than an isolated resonance. The overall continuum coupling of the series is, however, only redistributed. Consequently, the data of Fig. 4 exhibit a fast initial decay which is due to the broad $(n=1)$ resonance that overlaps with the resonances $n=2,3,4,5$.

In the present Letter, we reported the results of a timeresolved 2PPE investigation of image-potential resonances at metal surfaces for a situation where the coupling to the bulk continuum is strong enough to observe the effect of resonance trapping. Previous inverse photoemission and 2PPE experiments of aluminum surfaces lacked the energy resolution to distinguish resonances beyond $n=2$. For Al (100), a peak $0.2-0.5 \mathrm{eV}$ below vacuum level was observed by Veyan et al. [24], somewhat weaker but similar to that reported earlier for $\mathrm{Al}(111)$ [25-27]. Tentatively, this feature has been interpreted in terms of the $(n=1)$ imagepotential resonance or the superposition of a broadened full series of resonances $[17,28,29]$. In view of the present experiments and the model calculations performed for different coupling strengths (cf. Fig. 3), it appears likely that a reinspection of $\mathrm{Al}(111)$ with better resolution should reveal qualitatively similar results as reported here for $\mathrm{Al}$ (100), i.e., a spectrum with the most prominent peak originating from the $(n=2)$ resonance. Theoretically, it will be most interesting to see whether the exact treatment of a jellium surface, i.e., the case of infinitely strong coupling, leads to well-resolved resonances $n \geq 2$ or to one broad maximum in the surface density near the position of the $(n=1)$ state [17].

In conclusion, we have spectrally resolved the imagepotential resonances at the surface of a simple metal, i.e., $\mathrm{Al}(100)$, and investigated their decay into the bulk metal with time-resolved 2PPE. Despite a very strong coupling to the continuum, a clearly separated series of resonances could be observed for quantum numbers $n \geq 2$, whereas the $(n=1)$ resonance was found to be suppressed. The results are explained in terms of resonance trapping, which sets in for broadening that approaches the level spacings. Since similar situations of strongly coupled, but energetically close, resonances can frequently occur at surfaces, for molecular adsorbates, e.g., or for clusters, we believe that the experimental and theoretical investigation of such resonance phenomena deserves further attention.

It is a pleasure to thank P. M. Echenique, J. Güdde, and P. Thomas for valuable discussions, as well as N. Armbrust and M. Marks for help in setting up the experiment. We gratefully acknowledge funding by the Deutsche Forschungsgemeinschaft through GK 790 as well as support by the Ikerbasque Foundation.

[1] Dynamics at Solid State Surfaces and Interfaces, edited by U. Bovensiepen, H. Petek, and M. Wolf (Wiley-VCH, Berlin, 2010).

[2] P. M. Echenique, R. Berndt, E. V. Chulkov, Th. Fauster, A. Goldmann, and U. Höfer, Surf. Sci. Rep. 52, 219 (2004).

[3] A. G. Borisov, J.P. Gauyacq, A. K. Kazansky, E. V. Chulkov, V. M. Silkin, and P. M. Echenique, Phys. Rev. Lett. 86, 488 (2001).

[4] A. Föhlisch, P. Feulner, F. Hennies, A. Fink, D. Menzel, D. Sanchez-Portal, P. M. Echenique, and W. Wurth, Nature (London) 436, 373 (2005).

[5] X. Y. Zhu, Surf. Sci. Rep. 56, 1 (2004).

[6] H. Feshbach, Ann. Phys. (N.Y.) 5, 357 (1958).

[7] U. Fano, Phys. Rev. 124, 1866 (1961).

[8] M. Desouter-Lecomte and V. Jacques, J. Phys. B 28, 3225 (1995).

[9] C. Jung, M. Müller, and I. Rotter, Phys. Rev. E 60, 114 (1999).

[10] Th. Fauster and W. Steinmann, in Electromagnetic Waves: Recent Developments in Research, edited by P. Halevi (North-Holland, Amsterdam, 1995), Vol. 2, p. 347.

[11] U. Höfer, I. L. Shumay, C. Reuß, U. Thomann, W. Wallauer, and Th. Fauster, Science 277, 1480 (1997).

[12] R. L. Lingle, N.-H. Ge, R. E. Jordan, J. D. McNeill, and C. B. Harris, Chem. Phys. 205, 191 (1996).

[13] M. Wolf, E. Knoesel, and T. Hertel, Phys. Rev. B 54, R5295 (1996).

[14] M. Weinelt, J. Phys. Condens. Matter 14, R1099 (2002).

[15] P. M. Echenique and J. B. Pendry, J. Phys. C 11, 2065 (1978).

[16] A. G. Borisov, E. V. Chulkov, and P. M. Echenique, Phys. Rev. B 73, 073402 (2006).

[17] G. Fratesi, G. P. Brivio, P. Rinke, and R. W. Godby, Phys. Rev. B 68, 195404 (2003).

[18] H. J. Levinson, F. Greuter, and E. W. Plummer, Phys. Rev. B 27, 727 (1983).

[19] E. E. Krasovskii, W. Schattke, P. Jiříček, M. Vondráček, O. V. Krasovska, V. N. Antonov, A. P. Shpak, and I. Bartoš, Phys. Rev. B 78, 165406 (2008). 
[20] The calculated densities of states of Fig. 3 should only be compared qualitatively with the measured 2PPE intensities (Fig. 1). In addition to an experimental broadening of the more narrow higher resonances (mainly due to the finite bandwidth of the short laser pulses), different matrix elements for the population and photoemission of the resonance can change their relative heights. Moreover, interference with continuum excitations can distort and shift the observed resonance lines [7,21].

[21] Ch. Eickhoff, M. Teichmann, and M. Weinelt, Phys. Rev. Lett. 107, 176804 (2011).

[22] The 10 first levels of the Rydberg series and 5500 continuum states between $-3 \mathrm{eV}$ and $2.5 \mathrm{eV}$ have been included in the numerical simulations. In order to minimize the number of parameters, we neglect true dephasing, as well as inelastic decay, due to electron-hole-pair excitation. We assume a $n^{-3 / 2}$ scaling of the matrix elements of the Rydberg states [23]. For the proper description, near zero delay, we take into account the
IR-pump and UV-probe 2PPE process but do not consider the possibility of bulk excitations.

[23] T. Klamroth, P. Saalfrank, and U. Höfer, Phys. Rev. B 64, 035420 (2001).

[24] J. F. Veyan, W. Ibañez, R. A. Bartynski, P. Vargas, and P. Häberle, Phys. Rev. B 71, 155416 (2005).

[25] D. Heskett, K.-H. Frank, E. E. Koch, and H.-J. Freund, Phys. Rev. B 36, 1276 (1987).

[26] S. Yang, R. A. Bartynski, G. P. Kochanski, S. Papadia, T. Fondén, and M. Persson, Phys. Rev. Lett. 70, 849 (1993).

[27] V. Bulović, B. Quiniou, and R. M. Osgood, J. Vac. Sci. Technol. A 12, 2201 (1994).

[28] S. Å. Lindgren and L. Wallden, Phys. Rev. B 40, 11546 (1989).

[29] S. Papadia, M. Persson, and L.-A. Salmi, Phys. Rev. B 41, 10237 (1990).

[30] M. Heinrichsmeier, A. Fleszar, W. Hanke, and A. G. Eguiluz, Phys. Rev. B 57, 14974 (1998). 